\title{
Synote Second Screening: Using Mobile Devices for Video Annotation and Control
}

\author{
Mike Wald, Yunjia Li, George Cockshull, David Hulme, Douglas Moore, Aidan \\ Purdy-Say, James Robinson
}

ECS, University of Southampton, Southampton, UK

m.wald@soton.ac.uk

\begin{abstract}
This paper describes a new important enhancement to Synote, the freely available, award winning, open source, web based application that makes web hosted recordings easier to access, search, manage, and exploit for learners, teachers and other users. The feature supports 'flipped' classrooms and allows students to ask questions through annotations on their personal mobile devices while also being able to remotely control and play relevant video fragments.
\end{abstract}

Keywords: second screen, recorded lectures, learning, flipping classroom

\section{Introduction}

In their study of eLearning tools Rohani and Yazdani [1] identified several issues including a lack of social networking, a failure to use tags to categorise resources and an inability to raise questions or share comments. The award winning web application Synote helps address these issues as captioned video can be tagged and users can add comments as accessible synchronised annotations [2]. Text, audio, images, annotation and video can enhance the effectiveness of conveying information through eLearning [3] and a recorded lecture allows a student to decide when and where they wish to study. At home students can work at their own pace taking regular breaks to reduce fatigue and can play a video with their preferred screen size, audio volume, lighting and assistive technologies, pausing or rewinding when necessary to make it easier for them to take notes. 'Flipping the Classroom' (3) means teachers can ask students to watch videos at home and then discuss them in class afterwards. Students finding some of the content difficult to understand may not actually ask the questions in class that they had prepared at home due to communication difficulties, lack of confidence, or time pressures. Synote Second Screening helps address these issues by allowing students to ask questions through annotations on their personal devices while also being able to remotely control and play the relevant video fragments. The lecturer or the other students in the room can use media fragment annotations to respond to these questions or post additional information about the video. Kam et al. [4] showed that 
$66 \%$ of users preferred collaborative to independent note taking and Synote Second Screening could help facilitate this.

\section{Example Use Case}

Synote Second Screening enables annotations to be made in real time on mobile second screen devices when a video is replayed on a main screen. Students using controls on their mobile device can navigate through the presenter's video while being able to add annotations to the video for a chosen duration with an optional fragment added to highlight a particular region of the video. The ability to control a video from a second screen can be disabled by the presenter. All text and image thumbnail annotations are available on each participant's second screen. Thumbnails alt attributes are automatically made relevant and correct for the image, so a screen reader will announce "The thumbnail for [video name] at [timecode]". Synote Second Screening promotes interactive, collaborative working in real-time and allows reviewing of annotations from previous sessions.

Participants are undergraduate geologists who are beginning to learn about Hydraulic Fracking. They were asked by their lecturer, Laura to watch half of an introductory BBC Documentary video at home and are now discussing it in class. Figure 1 shows John's tablet in front of the main screen as he joins the new discussion session with Description 'Review of BBC Hydraulic Fracking Documentary' and Location '46/3001'. Figure 1 also shows that before John joins the session Laura is the only participant and she has selected the three options: 'show annotations from other sessions" which makes annotations added in any previous sessions also visible in this session; 'allow participants to control video player' so they can show Laura which media fragments they wish to discuss; 'pin annotations to player timeline' to give a quick overview on the timeline of which parts of the video have been annotated and whether they are important and questions or comments.

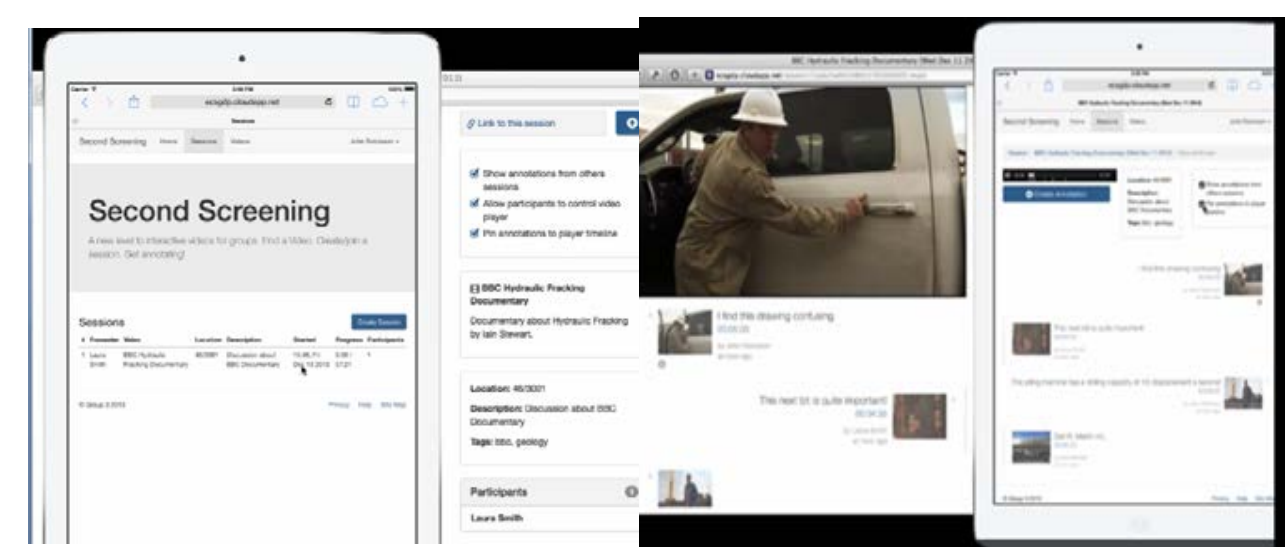

Fig. 1. Laura creates session from tablet fragment on main screen

Fig. 2. John selects timecode toplay video 

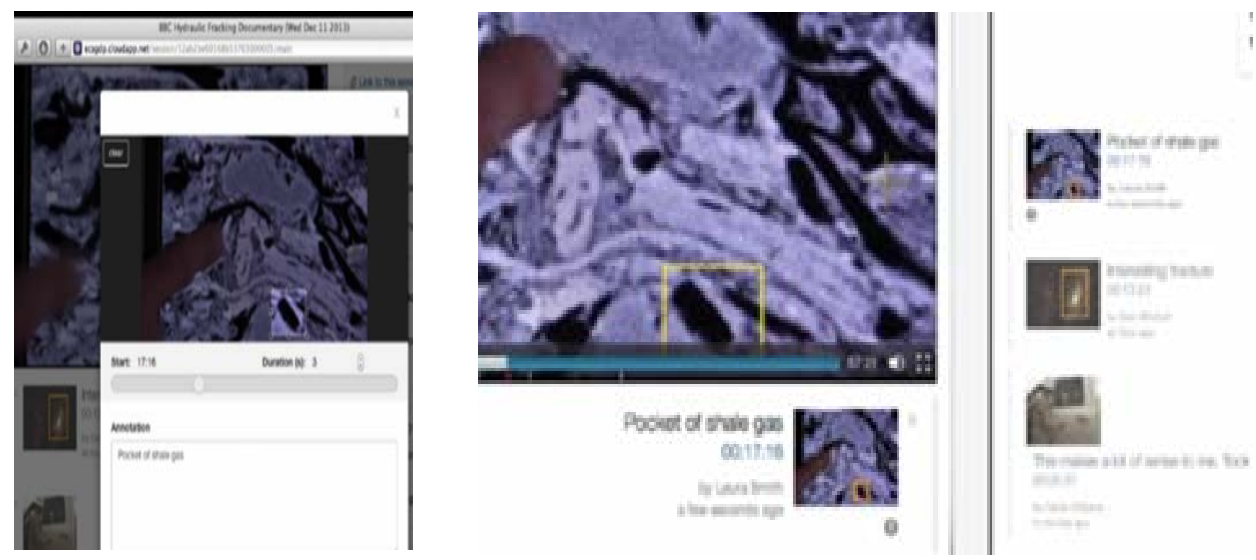

Fig. 3. Laura marks media fragment and flags as important

Fig. 4. Fragment on main screen video and timeline pips

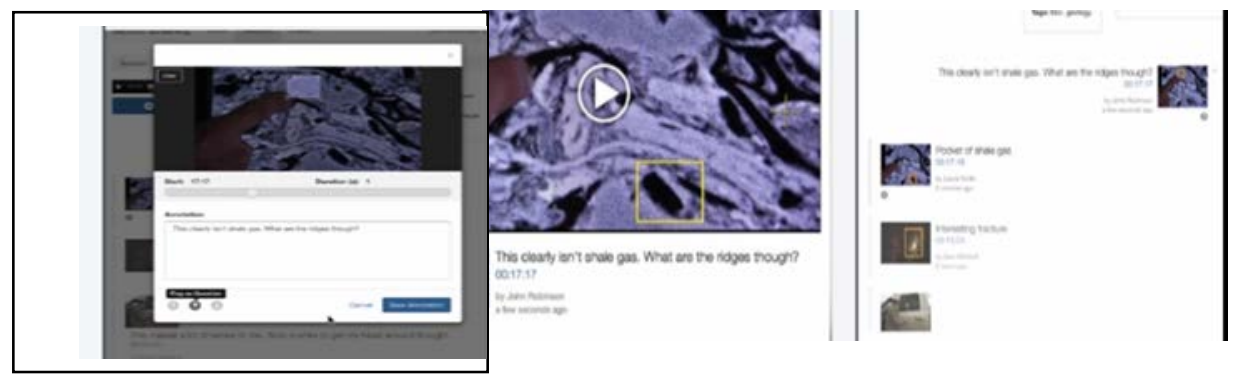

Fig. 5. John selects area, writes annotation Fig. 6. John's annotation and fragment thumbnail and flags as question appears on all screens

All participants now join the session by selecting from the session list. Laura gives an overview of the video, and then asks the participants if they have any questions. John explains he had an issue understanding a part of the explanation of the fracking process. John had created an annotation from 5:03-5:26 at home stating that he found it confusing. He selects the time code on this annotation to play this video fragment on the main screen (Figure 2). The video starts at 5.03 and stops automatically at 5.26. Laura is then able to explain the fracking process in more detail and to John's satisfaction before asking if there are any more questions. Sarah asks Laura to explain the image of rock more thoroughly and uses the timeline on her device to jump to the appropriate point in the video which is 17:46 to 17:49. Laura draws a rectangular fragment on a dark pore region and explains that this is where the gas collects and flags it as important (Figure 3). Laura continues the explanation and replays the video which now shows the marked fragment as a yellow rectangle with the importance of the annotation shown on the timeline by pip colour (Figure 4). John then 
creates the annotation 'this clearly isn't Shale Gas, what are the ridges though?' with a fragment on a light region of the thumbnail image and flags it as a question (Figure 5) and it appears on all screens as a yellow rectangle (Figure 6). When the class ends and Laura signs out on the main screen an alert box on John's second screen shows that the session has ended.

\section{$3 \quad$ Testing and Evaluation}

The final version of Synote Second Screening was developed following evaluation and comment on an initial prototype by a focus group of seven students. PowerMapper and Webaim's Wave toolbar for Firefox and The Paciello Group Colour Contrast Analyser were used to help confirm accessibility. Testing and user evaluations took place through 10 participants simulating real classroom tasks using keyboard and mouse input on laptops and touchscreen input on tablets and smartphones. The tasks were: Joining a session; Adding an annotation; Adding a media fragment; Marking a new annotation as a question; Deleting a personal annotation; Controlling the video playback; Playing a media fragment; Viewing session comments only and Creating a session. Analysis of results showed that Synote Second Screening was accessible, effective and intuitive to use and $80 \%$ of participants agreed the system would help aid in collaborative note taking and $90 \%$ of participants said that they would want to use the system in classes.

\section{Conclusion and Future Work}

Students would welcome the use of Synote Second Screening in classrooms and it would help improve accessibility, engagement and learning. Planned future work includes: the addition of a 'one-click' button to notify a lecturer that something in the video was confusing; a more advanced, permission-based authorisation system to enable the teacher to allow only certain participants to control playback from their second screen device; providing a text box to search for a timecode rather than only moving the timeline slider to a specific location in a video; and classrooms trials.

\section{References}

1. Rohani, M. B., Yazdani, K. Web 2.0 Embedded E-Learning : A Case Study. International Conference on Innovation Management and Technology Research (ICIMTR), 2012, pp. 603-608.

2. Wald, M Important new enhancements to inclusive learning using recorded lectures. In, 13th International Conference on Computers Helping People with Special Needs (ICCHP 2012), Linz, AT,11 - 13 Jul 2012. 8pp

3. Wallace, A. Social learning platforms and the flipped classroom. Second International Conference on e-Learning and e-Technologies in Education (ICEEE), 2013, pp. 198- 200

4. Kam, M., Wang, J., Iles, A., Tse, E., Chiu, J., Glaser, D., Tarshish, O., Canny, J. Livenotes: a system for cooperative and augmented note-taking in lecture. Proceedings of the SIGCHI conference on Human factors in computing systems - CHI '05, 2005 pp. 531- 540 